\title{
The natural history of ulcerative colitis in a pediatric population: a follow-up population- based cohort study
}

This article was published in the following Dove Press journal:

Clinical and Experimental Gastroenterology

14 June 2013

Number of times this article has been viewed

\author{
Hoda M Malaty ${ }^{1,2}$ \\ Bincy P Abraham ${ }^{1,3}$ \\ Seema Mehta ${ }^{2,3}$ \\ Elizabeth A Garnett ${ }^{4}$ \\ George D Ferry ${ }^{2,3}$ \\ 'Department of Medicine, \\ ${ }^{2}$ Department of Pediatrics, ${ }^{3}$ Texas \\ Children's Hospital, Baylor College \\ of Medicine, Houston, TX, USA \\ ${ }^{4}$ University of California, San \\ Francisco, CA, USA
}

Background: The natural history of ulcerative colitis (UC) has been poorly studied in children.

Methods: We performed a retrospective study in children diagnosed with UC with a follow-up. The diagnosis of UC was based on clinical, radiologic, endoscopic, and histologic examinations. We estimated the occurrence of colectomy, proctitis, and extraintestinal manifestations (EIMs) at the onset of the diagnosis and at the end of the study period.

Results: We identified 115 UC patients between 1986 and 2003 with a mean age at diagnosis of $10.6 \pm 5.1$ years. The cumulative rate of colectomy was $4.1 \%$ at 1 year, and $16 \%$ at 10 years. EIMs were experienced by $20 \%$ of the children; $48 \%$ had arthritis, $35 \%$ had sclerosing cholangitis, and $17 \%$ had aphthous stomatitis. Proctitis was noted in 29 patients and it was not associated with an increased risk of colectomy (relative risk $=1.4 ; 95 \% \mathrm{CI}=0.7-4.5$ ), and girls were twice more likely to develop proctitis. The pathologic reading for disease extensions was recorded for all children at entry and only 62 children had pathological results at maximum follow-up. At entry, $25 \%$ of the children only had ulcerative proctitis (E1) localization, $40 \%$ had left-sided UC (E2), and 35\% had extensive UC (E3). Among the patients with E1 localization, 20\% had progressed to $\mathrm{E} 2$ and $80 \%$ had progressed to E3; among the patients with E2 localization, $40 \%$ had progressed to E3. Age, gender, and EIMs at time of diagnosis were not associated with extension of disease at maximal follow-up. The $\mathrm{Z}$ score of body mass index (BMI) of children was significantly higher at the end of the study. At diagnosis, $85 \%$ of patients received 5 -aminosalicyclic acid, $60 \%$ received steroids, and $11 \%$ received an immunomodulator. The majority of patients were still using systemic steroids at and after 5 years from their entry date. Only 32 of the 91 children on steroids did not receive an immunomodulator.

Conclusion: Pediatric UC is associated with high rates of EIMs and colectomy that are not dependent on age, gender, or race, but is associated with a high rate of proctitis among girls. Understanding the clinical course of UC can optimize therapeutic interventions.

Keywords: inflammatory bowel disease, ulcerative colitis, children, natural history

\section{Introduction}

The epidemiology of inflammatory bowel diseases (IBD) has been well studied in adults; however, information regarding children is still scanty. Understanding the epidemiology of IBD in pediatrics is required to comprehend the natural history of the disease. Several studies have shown rising incidence of pediatric Crohn's disease (CD) over the last several years; however, the incidence of ulcerative colitis (UC) has remained stable or marginally decreased..$^{1-9} \mathrm{UC}$ disease has an early onset; children could be at high risk of disease complications, surgery, and develop extraintestinal
Correspondence: Hoda M Malaty Veterans Affairs Medical Center (I I ID), 2002 Holcombe Blvd, Houston,

Texas 77030, USA

$\mathrm{Tel}+\mathrm{I} 7137950232$

Fax +I 7137901040

Email hmalaty@bcm.tmc.edu 
manifestations (EIMs). Therefore, there is a great need to study the natural history of UC to optimize therapeutic management and intervention.

It has been recommended to use mesalamine for treating $\mathrm{UC}$ in the pediatric population as the first course of action before moving onto immunomodulators or biologics. ${ }^{10-12}$ Until today, the natural history of UC in children is not extensively studied. ${ }^{13,14}$ Children with UC represent a unique cohort of patients to investigate because of the initial host immune response, need for early intervention, and the natural history of the disease. ${ }^{15-18}$ Therefore, we aimed to study the natural history of UC among children to characterize the clinical course of the disease and to identify possible factors associated with surgery, EIMs, proctitis, and use of current therapy in a population-based pediatric cohort identified in the IBD Registry at the Texas Children's Hospital (TCH). Because of the unknown etiology of the disease, there is a great need in the pediatric population to examine the predictive factors in the course of UC that can be used to improve therapeutic interventions.

\section{Materials and methods Patient population}

A retrospective epidemiologic investigation was conducted to identify a cohort of children diagnosed with IBD and registered in the IBD Center at TCH. The hospital is located in the Greater Houston Metropolitan Area which consists of ten counties with a diverse racial and ethnic population of 5.5 million. ${ }^{19,20}$ Our study included children with a confirmed diagnosis of IBD.

A cohort of children diagnosed with UC was studied from the period of 1986 to 2003. Cases were identified through a computer program that generated the database for the studied cohort. UC diagnoses were based on clinical, radiological, endoscopic, and histological criteria. ${ }^{21,22}$ The IBD registry obtained institutional review board approval for the protocol. Informed consent and assent were obtained from parents and patients before entry of patient's information into the registry.

\section{Data collection}

Demographic data obtained included month of birth, gender, and race/ethnic background. Anthropomorphic data (height and weight) were documented for each visit and corresponding body mass indices (BMIs) were determined. BMI/age and height/age $\mathrm{Z}$ scores were calculated. Medications reviewed included the following: azulfidine, 5-aminosalicylic acids ([5-ASA] oral, enema, or suppository), steroids (oral prednisone or prednisolone), antibiotics (eg, metronidazole), immunomodulators (azathioprine [AZA], 6-mercaptopurine, methotrexate, and cyclosporine), and infliximab. Patients were classified as having been exposed to medication if it was started at any time from diagnosis or during the follow-up period.

\section{Outcome measures/variables descriptions}

Disease diagnosis and distribution were identified based on a review of all endoscopy, pathology, and radiology records. We estimated the occurrence of colectomy, presence of proctitis, and EIMs at the onset of the diagnosis and at the end of the study period. EIMs were defined as arthritis, aphthous stomatitis, arthralgia, erythema nodosum, skin lesions, and/or primary sclerosing cholangitis. Diagnosis of EIMs was decision of the specialist physician at TCH. UC localization at diagnosis and maximal follow-up was classified according to the Montreal classification. ${ }^{23}$ Ulcerative proctitis (E1) was defined as involvement limited to the rectum (ie, proximal extent of inflammation distal to the rectosigmoid junction). Left-sided UC (E2) was defined as involvement limited to the portion of the colorectum distal to the splenic flexure. Extensive UC (E3) was defined as involvement extending proximally to the splenic flexure. Osteoporosis/osteopenia was counted as disease outcome for UC and was diagnosed based on dual energy X-ray absorptiometry (DEXA) scan.

\section{Statistical analysis}

Each patient's initial visit was logged as their enrollment visit and all subsequent visits were logged as follow-up visits. Patients with no follow-up visits or one follow-up visit that occurred less than 1 year after enrollment were excluded. The cumulative risk of colectomy was estimated by the Kaplan-Meier method, and risk factors for colectomy were estimated with Cox hazards proportional models. The 95\% confidence intervals (CIs) were calculated, assuming a Poisson distribution of cases.

The time interval between the initial and final visits was determined and the corresponding change in the BMI and height $\mathrm{Z}$ scores was calculated. Paired and unpaired $t$-tests were used to compare BMI and height $\mathrm{Z}$ scores.

\section{Results}

\section{Population characteristics of UC (1986 to 2003)}

Between 1986 and 2003, 120 children had UC, with a median follow-up time of $4.4 \pm 2.1$ years. Five patients diagnosed with UC were lost to follow-up. Among the remaining 
UC patients, 115 had a follow-up time equal to or greater than 2 years and included 52 males and 63 females (male: female ratio of $0.8: 1$ ), with a mean age at diagnosis of $10.6 \pm 5.1$ years with no significant difference between boys and girls $(8.4 \pm 5.3$ years and $10.7 \pm 5.0$ years, respectively, $P=0.81)$. There were $92(80 \%)$ Caucasians and the other $20 \%$ were divided between Hispanics, African Americans, and Asians.

\section{Extraintestinal manifestations (EIMs)}

Twenty three patients (20\%) experienced one or more EIMs of whom eleven (48\%) were diagnosed with arthritis (axial, peripheral, or both), eight (35\%) had sclerosing cholangitis, and four (17\%) had aphthous stomatitis. Among children with EIMs, 14 (61\%) patients had EIMs at baseline, nine (39\%) patients developed EIMs during the follow-up period, and three (13\%) patients had more than one EIM. Because of the controversy of inclusion of osteopenia/osteoporosis as EIMs, they were excluded at the study entry and were considered as postdiagnostic EIMs. There were four children diagnosed with osteoporosis or osteopenia after the onset of diagnosis of UC (3.5\%). EIMs were not associated with age or gender; however, children with EIMs had longer duration of follow-up than those without EIMs $(5.2 \pm 2.6$ years versus $3.1 \pm 2.2$ years, respectively).

\section{Proctitis and disease extension}

From the initial visit to the end point of the study, proctitis was noted in 29 patients ( $25 \%$ ) and it was not associated with an increased risk of colectomy $(\mathrm{OR}=1.4 ; 95 \% \mathrm{CI}=0.7-4.5)$. A significant gender difference was noted as almost twice as many girls were diagnosed with proctitis than boys $(30 \%$ versus $17 \%$, respectively, $P=0.043$ ). The mean age at diagnosis of proctitis was $10.1 \pm 5.6$ years and girls tended to be older when diagnosed with proctitis then boys (mean age of $10.6 \pm 5.8$ years versus $8.5 \pm 5.2$ years, respectively, $P=0.062$ ).

The pathologic reading for disease extensions was recorded for all children at entry and only 62 children had pathological results at maximum follow-up. At entry, $25 \%$ of children had E1 localization, 40\% had E2, and 35\% had E3. Among patients with E1 localization, 20\% had progressed to $\mathrm{E} 2$ and $80 \%$ had progressed to E3; among patients with E2 localization, $40 \%$ had progressed to E3. Age, gender, and EIMs at time of diagnosis were not associated with extension of disease at maximal follow-up (OR $=1.3,95 \%$ $\mathrm{CI}=0.3-4.2, P=0.54 ; \mathrm{OR}=1.3,95 \% \mathrm{CI}=0.4-3.2, P=0.65$; and $\mathrm{OR}=1.1,95 \% \mathrm{CI}=0.4-2.8, P=0.81$, respectively).

\section{Colectomy}

Twenty patients underwent surgery at least once during the study period (six patients had partial colectomy and 14 had total colectomy), resulting in a crude colectomy rate of $17 \%$. In a Kaplan-Meier analysis, the probability of colectomy significantly increased from $4.1 \%$ at 1 year to $16 \%$ at 10 years (Figure 1). In univariate analysis, EIMs at diagnosis were not significantly associated with increased risk of need for surgery $(\mathrm{OR}=1.4 ; 95 \% \mathrm{CI}=0.3-4.0)$. Boys were two times more likely to undergo colectomy than girls $(\mathrm{OR}=2.1$, 95\% CI $=1.1-8.2, P=0.04)$. Age, time between onset of symptoms, and diagnosis were not associated with surgery.

\section{$\mathrm{BMI}$}

We characterized the growth pattern of children with UC at both their initial presentation and maximal follow-up. The mean height $\mathrm{Z}$ score for children with UC decreased slightly from the initial to final visit $(-0.11 \pm 1.14$ versus $-0.17 \pm 1.12$, $P=0.35)$. However, the mean BMI $\mathrm{Z}$ score increased from $-0.04 \pm 1.43$ to $0.31 \pm 1.07(P<0.001)$.

\section{Medical treatment received at initial and at maximal follow-up}

At entry, $85 \%$ of the patients received treatment with oral 5-ASA medications, $60 \%$ received oral steroids, $11 \%$ received immunomodulators (eg, 6-mercaptopurine/imuran), and 11\% received antibiotics. The use of antibiotics had doubled by the last follow-up period (Figure 2). Subanalysis revealed that the use of antibiotics was correlated to colectomy as many children with severe colitis who underwent colectomy were prescribed antibiotics.

The number of patients who received systemic steroids remained quite high as the majority of them were still using steroids at and after 5 years from the entry date. We further examined characteristics of patients on steroids and immunomodulators. Of the 91 subjects on steroids during the study period, 32 (35\%) did not receive an immunomodulator. However, only five of the 63 subjects $(8 \%)$ on an immunomodulator did not require steroids during the follow-up period. Rectal 5-ASAs were being prescribed at study entry and its use increased over the study period suggesting that these patients continued to have distal disease. None of the patients had received infliximab at the beginning of the study as the drug was approved for use in the US in 1998. Six patients received infliximab in 1998; one patient started in the first year from diagnosis, two in the second year, two in the fourth year, and one in the fifth year from diagnosis. 


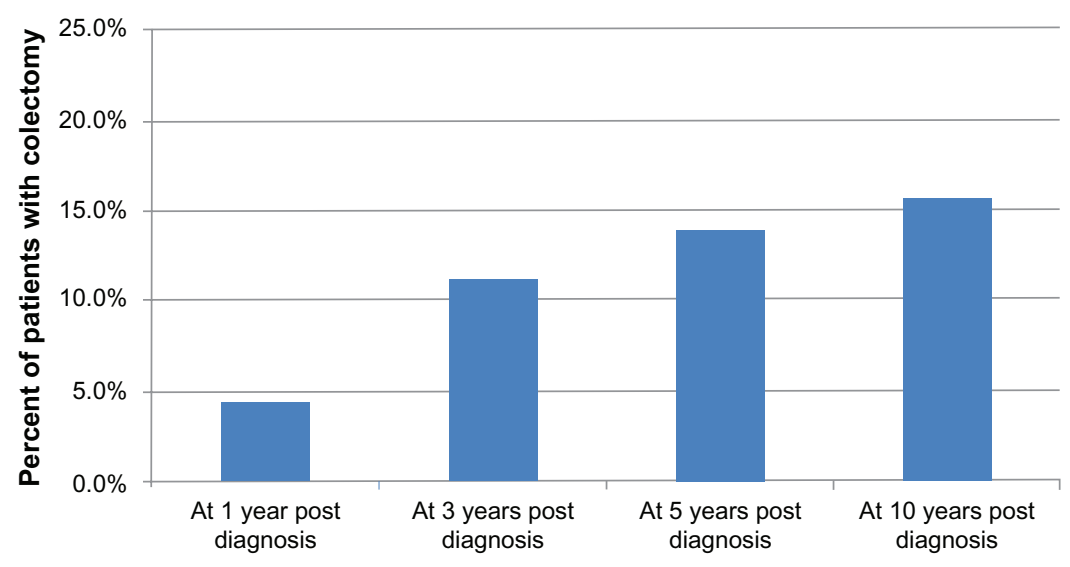

Figure I Postdiagnosis colectomy rate at I year, 3 years, 5 years, and 10 years among II 5 pediatric patients with ulcerative colitis.

\section{Discussion}

The main strength of this study is due to its populationbased design and the availability of detailed clinical information over time in the Pediatric IBD Registry. Diagnosis of UC was confirmed by colonoscopy and histological evaluation. All patients included in the analysis had at least 2 years of follow-up. Our findings suggest that the natural course of pediatric UC is severe for several reasons. First, the colectomy rate was $14 \%$ at 5 years and $16 \%$ at 10 years which reflects the severity of the cases in spite of therapies that led to the surgical intervention during the study period. However, our study was performed in the era before the introduction of biologic therapy as none of our subjects had received biologic therapy as a first line of therapy. Future studies are needed to address the effect of biologics on the rate of colectomy. Our results revealed that boys were twice more likely to have a colectomy as girls. It is possible that surgery in girls is often delayed or attempts are made to avoid it due to the risk of decreased fertility, especially with ileal pouch anal anastomosis. This finding was not consistent with Gower-Rousseau et al who reported no gender effect on the colectomy rate among a cohort of children with UC in France. ${ }^{14}$ Although both studies were conducted in pediatric populations and had comparable population size, the demographics and methods used varied.

Second, $20 \%$ of the studied children had one or more EIMs, and these mostly related to joint inflammation. These results are comparable to what was previously reported among UC children in the US. ${ }^{24-26}$ The study

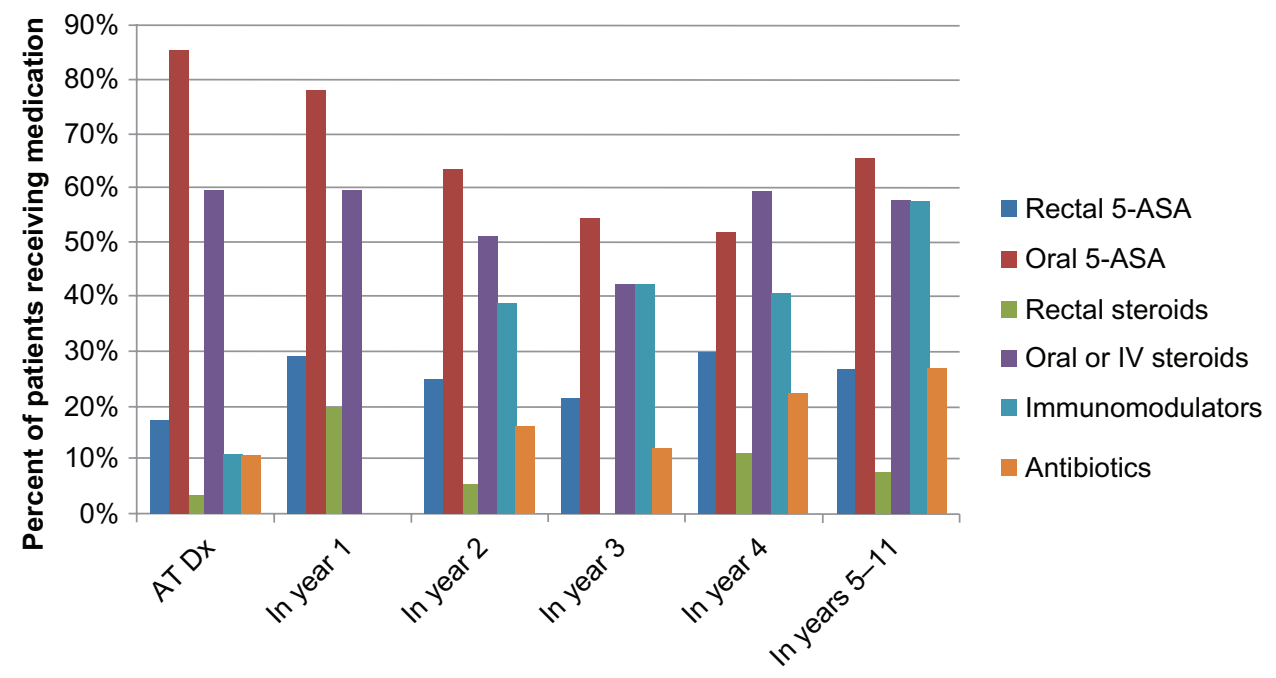

Figure 2 Treatments received at maximal follow-up in 115 pediatric patients with ulcerative colitis. Abbreviation: ASA, aminosalicylic acid. 
by Heyman et al reported 25\% EIMs in the total study population with no difference in the incidence of EIMs by IBD type. ${ }^{27}$ Our results revealed no age or race effect on the incidence of EIMs, which is consistent with other studies that reported no differences in symptom presentation and EIMs between Caucasians and African American pediatric patients. ${ }^{25,26}$ Moreover, we found that children with EIMs had longer duration of follow-up years than children without EIMs, potentially reflecting higher disease severity that prolonged the follow-up visits in this group. The results showed an incidence rate of $3.5 \%$ for abnormal bone density manifested as either osteopenia or osteoporosis post diagnosis of IBD. These could result from multiple factors including malabsorption of calcium and/or vitamin $\mathrm{D}$ (although less likely in UC compared to CD), steroid use, significant disease activity, and elevation of inflammatory cytokines. ${ }^{27-29}$ Although the pathogenesis of osteopenia/ osteoporosis is poorly understood in these patients, bone mineralization disorders can occur even without exposure to steroids. ${ }^{29}$

Of the studied children, $25 \%$ had proctitis during the study period and age had no effect on developing proctitis. This observation is not consistent with Heyman et al who reported that older patients with UC demonstrated more cases of proctitis than younger patients with UC. ${ }^{30}$ This difference could be due to a difference in locations of the studied population or a difference in the use of diagnostic methods for UC. It is still not clear why girls were two times more likely to have proctitis than boys. Since this observation was not previously reported, it deserves further investigation. The low proportion of proctitis in our study is evidence for the validity of our results as it has been long considered in the literature that the proportion of proctitis is a good marker for the completeness of collection of UC cases in epidemiological studies. ${ }^{31}$

Our findings of the initial extent of the disease were similar to that found in other pediatric studies. ${ }^{32,33}$ However, other recent studies have reported a higher rate $(>80 \%)$ of E3 location at diagnosis., ${ }^{2,34}$ This conflict in results could be because our study was population-based. Patients with less severe forms of UC may be seen in private care facilities rather than referred to academic centers. Another explanation is that the definition of disease extension in our study was based on microscopic evidence of inflammation rather than on macroscopic findings. ${ }^{2}$ The majority of UC cases progressed to more proximal parts or more extensive disease upon follow-up.
Our study did demonstrate a growth failure among the studied children at the time of the diagnosis. However, there was slight deterioration of the height $\mathrm{Z}$ score and slight improvement of the BMI by the end of follow-up period. Growth failure among IBD children has been previously reported in $\mathrm{CD}$, and occurs more frequently in $\mathrm{CD}$ than in $\mathrm{UC}$. In $\mathrm{UC}$, growth failure at diagnosis ranged from $0 \%$ to $10 \%$, while at follow-up this ranged from between $0 \%$ and $17 \% .{ }^{35-37}$ Therefore, growth failure should not be excluded for children with UC.

Almost all children in the studied cohort received 5-ASA at least once over the disease course, in accordance with the recent guidelines from the US and Europe that recommend 5-ASA as first line therapy in induction of response and remission, as well as in maintenance. ${ }^{10-12}$ This figure is similar to what was previously reported by Gower-Rousseau et al in France. ${ }^{14}$ At diagnosis and at 1 year, $60 \%$ of the studied cohort received systemic steroids, and this percentage remained high as the majority of patients were still using it even at 5 years from diagnosis. The use of immunomodulators was very low at diagnosis to the first year, but increased over the study period. Despite this increase in immunomodulator use, steroid use remained high as only $8 \%$ of children on immunomodulators did not take steroids during the follow-up period. Since the majority of the patients were on steroids, and $35 \%$ did not receive immunomodulators, it appears that these patients should be stepped up to immunomodulators to avoid complications of steroid use. The use of immunomodulators has increased more slowly in pediatric UC and its impact on the natural history of the disease remains difficult to assess. The antibiotic used for the current study was mainly Flagyl as it was commonly used for bad flares in conjunction with steroids.

The utilization of data for constructing a retrospective cohort has some shortcomings. Our study population was taken from a single pediatric hospital and there are possibilities that more severe cases are referred to academic centers. However, TCH is one of the largest children's hospitals in the nation and offers a diverse population that closely reflects that of the Texan population. Moreover, there are only two private pediatric gastroenterology practices, and these opened in the same area in 2000. Another limitation of the study is that the information on EIMs at diagnosis of IBD was retrospective and there were no standard criteria for the definition of each EIM. However, our definition for EIMs was based on what is reported in the literature. 
Additionally, we could not assess the impact of biologics on the course of UC due to the small number of patients who received infliximab. However, the study period did not allow this investigation as the drug was only introduced for clinical use in 1998. Finally, the missing information on disease severity and disease activity scores could have provided more insights to the natural history of the disease in children. The retrospective nature of the current epidemiologic study did not permit the analysis of these parameters. However, the analysis of the current variables over a long period of time provided several important findings regarding the natural history of UC in children.

This study demonstrates clear evidence that pediatric UC is associated with high rates of EIMs and colectomy which were not associated with age, gender, or race/ethnicity. It was also associated with a high rate of proctitis, especially among girls. The majority of UC cases progressed to more proximal parts or more extensive disease upon follow-up. It is possible that even within the same community different cohorts may have different risk factors for presenting with UC disease. It is important to study the natural history and the clinical course of UC in pediatrics during more recent years to compare the prognosis of the disease before and after the use of biologics.

\section{Acknowledgment}

This study was supported by a grant from Aptalis Pharma Canada Inc. The grantor had no input in the inception, conduct, analysis of the study, or writing of the content of the manuscript, except comments and reviews to the manuscript which the authors had no obligation to follow.

\section{Disclosure}

All the authors listed on the manuscript take full responsibility for the manuscript. There is no potential conflict of interest, real, or perceived on submitting that manuscript.

\section{References}

1. Malaty HM, Fan X, Opekun AR, Thibodeaux C, Ferry GD. Rising incidence of inflammatory bowel disease among children: a 12-year study. J Pediatr Gastroenterol. 2010;50:27-31.

2. Kugathasan S, Judd RH, Hoffmann RG, et al; Wisconsin Pediatric Inflammatory Bowel Disease Alliance. Epidemiologic and clinical characteristics of children with newly diagnosed inflammatory bowel disease in Wisconsin: a statewide population-based study. J Pediatr. 2003;143:525-531.

3. Russell MG. Changes in the incidence of inflammatory bowel disease: what does it mean? Eur J Intern Med. 2000;11:191-196.
4. Sawczenko A, Sandhu BK, Logan RF, et al. Prospective survey of childhood inflammatory bowel disease in the British Isles. Lancet. 2001;357:1093-1094.

5. Logan RF. Inflammatory bowel disease incidence: up, down or unchanged? Gut. 1998;42:309-311.

6. Loftus EV Jr. Clinical epidemiology of inflammatory bowel disease: incidence, prevalence, and environmental influences. Gastroenterology. 2004;6:1504-1517.

7. Turunen P, Kolho KL, Auvinen A, Iltanen S, Huhtala H, Ashorn M. Incidence of inflammatory bowel disease in Finnish children, 1987-2003. Inflamm Bowel Dis. 2006;12:677-683.

8. Lindberg E, Lindquist B, Holmquist L, Hildebrand H. Inflammatory bowel disease in children and adolescents in Sweden, 1984-1995. J Pediatr Gastroenterol Nutr. 2000;30:259-264.

9. Abraham B, Mehta S, El-Serag HB. Natural history of pediatric onset inflammatory bowel disease: a systematic review. J Clin Gastroenterol. 2012;46:581-589.

10. Baumgart DC. The diagnosis and treatment of Crohn's disease and ulcerative colitis. Dtsch Arztebl Int. 2009;106:123-133.

11. Ferry GD, Kirschner BS, Grand RJ, et al. Olsalazine versus sulfasalazine in mild to moderate childhood ulcerative colitis: results of the Pediatric Gastroenterology Collaborative Research Group Clinical Trial. J Pediatr Gastroenterol Nutr. 1993;17:32-38.

12. Baumgart DC, Sandborn WJ. Inflammatory bowel disease: clinical aspects and established and evolving therapies. Lancet. 2007;369: 1641-1657.

13. Benchimol EI, Fortinsky KJ, Gozdyra P, Van den Heuvel M, Van Limbergen J, Griffiths AM. Epidemiology of pediatric inflammatory bowel disease: a systematic review of international trends. Inflamm Bowel Dis. 2011;17:423-439.

14. Gower-Rousseau C, Dauchet L, Vernier-Massouille G, et al. The natural history of pediatric ulcerative colitis: a population-based cohort study. Am J Gastroenterol. 2009;104:2080-2088.

15. Leshinsky-Silver E, Karban A, Buzhakor E, et al. Is age of onset of Crohn's disease governed by mutations in NOD2/caspase recruitment domains 15 and toll-like receptor 4? Evaluation of a pediatric cohort. Pediatr Res. 2005;58:499-504.

16. Babusukumar U, Wang T, McGuire E, Broeckel U, Kugathasan S. Contribution of OCTN variants within the IBD5 locus to pediatric onset Crohn's disease. Am J Gastroenterol. 2006;101:1354-1361.

17. Griffiths AM. Specificities of inflammatory bowel disease in childhood. Best Pract Res Clin Gastroenterol. 2004;18:509-523.

18. Bousvaros A, Sylvester F, Kugathasan S, et al; Challenges in Pediatric IBD Study Groups. Challenges in pediatric inflammatory bowel disease. Inflamm Bowel Dis. 2006;12:885-913.

19. The City of Houston. Planning and Development. Demographic Data. Available from: http://www.houstontx.gov/planning/Demographics/ demog_links.html. Accessed April 30, 2013.

20. The City of Houston. Demographic Data. Available from: http://www. houstontx.gov/planning/Demographics/demog_descriptions.html. Accessed April 30, 2013.

21. Kim SC, Ferry GD. Inflammatory bowel diseases in pediatric and adolescent patients: clinical, therapeutic, and psychosocial considerations. Gastroenterology. 2004;126:1550-1560.

22. Hyams J, Markowitz J, Otley A, et al; Pediatric Inflammatory Bowel Disease Collaborative Research Group. Evaluation of the pediatric Crohn's disease activity index: a prospective multicenter experience. $J$ Pediatr Gastroenterol Nutr. 2005;41:416-421.

23. Silverberg MS, Satsangi J, Ahmad T, et al. Toward an integrated clinical, molecular and serological classification of inflammatory bowel disease: Report of a Working Party of the 2005 Montreal World Congress of Gastroenterology. Can J Gastroenterol. 2005;19(Suppl A):5-36.

24. Jose FA, Garnett EA, Vittinghoff E, et al. Development of extraintestinal manifestations in pediatric patients with inflammatory bowel disease. Inflamm Bowel Dis. 2009;15:63-68. 
25. Eidelwein AP, Thompson R, Fiorino K, Abadon V, Oliva-Hemker M. Disease presentation and clinical course in black and white children with inflammatory bowel disease. J Pediatr Gastroenterol Nutr. 2007;44: 555-560.

26. White JM, O'Connor S, Winter HS, et al. Inflammatory bowel disease in African American children compared with other racial/ethnic groups in a multicenter registry. Clin Gastroenterol Hepatol. 2008;6: 1361-1369.

27. Heyman MB. Development of extraintestinal manifestations in pediatric patients with inflammatory bowel disease. J Pediatr Gastroenterol Nutr. 2009;48:243-245.

28. Vestergaard P. Prevalence and pathogenesis of osteoporosis in patients with inflammatory bowel disease. Minerva Med. 2004;95: 469-480.

29. Compston JE, Judd D, Crawley EO, et al. Osteoporosis in patients with inflammatory bowel disease. Gut. 1987;28:410-415.

30. Heyman MB, Kirschner BS, Gold BD, et al. Children with earlyonset inflammatory bowel disease (IBD): analysis of Pediatric IBD Consortium Registry. J Pediatr. 2005;146:35-40.

31. Mendeloff AI, Calkins B. The epidemiology of inflammatory bowel disease. In: Kirsner JB, Shorter RG, editors. Inflammatory Bowel Disease. Philadelphia, PA: Lea and Febiger; 1988;3-34.
32. Hyams JS, Davis P, Grancher K, Lerer T, Justinich CJ, Markowitz J. Clinical outcome of ulcerative colitis in children. J Pediatr. 1996;29: 81-88.

33. Barton JR, Ferguson A. Clinical features, morbidity and mortality of Scottish children with inflammatory bowel disease. Q J Med. 1990;75: 423-439.

34. Van Limbergen J, Russell RK, Drummond HE, et al. Definition of phenotypic characteristics of childhood-onset inflammatory bowel disease. Gastroenterology. 2008;135:1114-1122.

35. Ferguson A, Sedgwick DM. Juvenile onset inflammatory bowel disease: height and body mass index in adult life. BMJ. 1994;308: 1259-1263.

36. Ferguson A, Sedgwick DM. Juvenile-onset inflammatory bowel disease: predictors of morbidity and health status in early adult life. $J R$ Coll Physicians Lond. 1994;28:220-227.

37. Turunen P, Ashorn M, Auvinen A, Iltanen S, Huhtala H, Kolho KL. Long-term health outcomes in pediatric inflammatory bowel disease: a population-based study. Inflamm Bowel Dis. 2009;15:56-62.
Clinical and Experimental Gastroenterology

\section{Publish your work in this journal}

Clinical and Experimental Gastroenterology is an international, peerreviewed, open access journal, publishing all aspects of gastroenterology in the clinic and laboratory, including: Pathology, pathophysiology of gastrointestinal disease; Investigation and treatment of gastointestinal disease; Pharmacology of drugs used in the alimentary tract;

\section{Dovepress}

Immunology/genetics/genomics related to gastrointestinal disease. This journal is indexed on CAS. The manuscript management system is completely online and includes a very quick and fair peer-review system. Visit http://www.dovepress.com/testimonials.php to read real quotes from published authors.

Submit your manuscript here: http://www.dovepress.com/clinical-and-experimental-gastroenterology-journal 